\title{
The effects of simvastatin preconditioning on the expression of caspase-3 after myocardial ischemia reperfusion injury in rats
}

\author{
WEIXIN SUN ${ }^{1}$, RENYOU PAN $^{1}$, JUN SONG $^{1}$ and HONGLIN SUN ${ }^{2}$ \\ Departments of ${ }^{1}$ Cardiology and ${ }^{2}$ Pharmacy, Yancheng TCM Hospital Affiliated to Nanjing University \\ of Chinese Medicine, Yancheng, Jiangsu 224000, P.R. China
}

Received May 22, 2018; Accepted November 21, 2018

DOI: $10.3892 /$ etm.2019.7164

\begin{abstract}
Effect of simvastatin on the expression of caspase-3 in myocardial ischemia reperfusion injury in rats was observed to explore the protective effect of caspase- 3 through anti-apoptosis mechanism. A total of 48 healthy male SD rats weighing 160-240 g were selected and divided into 4 groups randomly, namely, the blank group, the sham operation group, the ischemia-reperfusion group and the simvastatin group, with 12 rats in each group. The model of SD rats was made by ligation. The loosen ligature made the reperfusion animal model, the occurrence of arrhythmia in the electrocardiogram of lead II in the experimental animal model was observed, and the area of myocardial infarction in the experimental animal models was detected. The number of apoptotic cells was detected by immunohistochemistry, and the expression of caspase-3 was detected by western blotting. The infarct area in the simvastatin group was significantly lower than the ischemia reperfusion group $(\mathrm{P}<0.05)$. The positive rate of the expression of caspase- 3 and the positive rate of the expression of apoptotic cells in the ischemic reperfusion and simvastatin groups were significantly higher than that of the blank and sham operation groups, and the positive rate of the expression of caspase- 3 and apoptotic cells in the simvastatin group was significantly lower than that of the ischemia-reperfusion group $(\mathrm{P}<0.05)$. The arrhythmia score of the simvastatin group was significantly lower than that of the ischemia-reperfusion group $(\mathrm{P}<0.05)$. Compared with the blank and sham operation groups, the expression of caspase-3 protein in the ischemia-reperfusion and simvastatin groups was significantly increased, and the expression of caspase-3 protein in the simvastatin group was significantly lower than that of the ischemia reperfusion group $(\mathrm{P}<0.05)$. Simvastatin has a protective effect on myocardial ischemia-reperfusion injury, which may be related to the reduction of caspase-3 expression and inhibition of apoptosis.
\end{abstract}

Correspondence to: Mr. Honglin Sun, Department of Pharmacy, Yancheng TCM Hospital Affiliated to Nanjing University of Chinese Medicine, 53 Renmin Middle Road, Yancheng, Jiangsu 224000, P.R. China

E-mail: honglin_sun@163.com

Key words: simvastatin, ischemia reperfusion injury, caspase-3

\section{Introduction}

Statins are HMG-CoA reductase inhibitors. There are many statins in the market, such as simvastatin, lovastatin, pravastatin and simvastatin (1). It has been confirmed that statins can make HMG-CoA unable to convert to mevalonic acid which is a necessary substance for the synthesis of cholesterol, which eventually leads to the inhibition of cholesterol synthesis in the body (2). Furthermore, it has been found that after preconditioning with pravastatin, it can effectively inhibit apoptosis of renal ischemia reperfusion injury, and has a better effect on renal protection (3). Statins are also becoming increasingly prominent in the prevention and treatment of heart disease. Necrosis in the ischemic central region is the main manifestation of myocardial ischemia and reperfusion, and there will be obvious apoptosis in the semi-dark zone (4). In this study, an animal model of simvastatin preconditioning myocardial ischemia reperfusion injury is used to observe the effect of the expression of apoptosis inhibitor caspase-3.

\section{Materials and methods}

Animals and groups. A total of 48 healthy male SD rats aged 8 weeks and weighing 160-240 g were purchased from Laboratory Animal Center of Sichuan University (Chengdu, China), randomly numbered and divided into 4 groups, namely, the blank group, the sham operation group, the ischemia-reperfusion group and simvastatin group with 12 rats in each group. The feeding environment of the rats was of SPF grade. The ambient temperature was $22 \pm 1^{\circ} \mathrm{C}$ and the ambient humidity was $45 \pm 3 \%$. The rats in each group could eat and drink freely according to the circadian rhythm.

The study was approved by the Ethics Committee of Yancheng TCM Hospital Affiliated to Nanjing University of Chinese Medicine (Yancheng, China). All the experimental operations involved in this experiment were carried out in accordance with the relevant provisions of the NIH guidelines for the use of laboratory animals.

Reagent consumables. Simvastatin was purchased from Shanghai Yanjing Biological Technology Co., Ltd. (Shanghai, China), nitro tetrazolium chloride (NBT) was purchased from Shanghai Haoran Bio Technologies Co., Ltd. (Shanghai, China), caspase-3 rabbit polyclonal antibody was purchased 
from Cell Signaling Technology, Inc. (Danvers, USA; cat. no. 9662), and SABC immunohistochemical staining kits were purchased from Shanghai S\&S Bio \& Tech Co., Ltd. (Shanghai, China; http://www.ssmotor-sh.com/), upgraded packing of one step TUNEL apoptosis in situ assay kits were purchased from Jiangsu Keygen Biotech Co., Ltd. (Jiangsu, China). ECL luminescent liquids was purchased from Cell Signaling Technology Inc.).

Administration. SD rats in the blank group were reared normally. Simvastatin was compounded into suspension state by using medical saline. The amount of $20 \mathrm{mg} / \mathrm{kg}$ was administered to SD rats at ten days before operation, once a day at the same time, the same volume of isosmotic saline was used for ten days in the sham operation and ischemia reperfusion groups, once a day at the same time.

Animal model of ischemia-reperfusion injury. According to the method provided by Hadi et al (5), ischemia reperfusion model was established. The 4 groups of SD rats were fasted for $12 \mathrm{~h}$ before the model was built. In addition to the blank group, all the SD rats in all the other groups were anesthetized with $10 \%$ concentration of chloral hydrate. The limbs of the $\mathrm{SD}$ rats were fixed on the operating table. The rat chests were disinfected by medical alcohol after hair removal, the trachea was cut by the surgical blade, using mechanical auxiliary ventilation and the chest was opened along the left edge of the sternum to expose the heart position of the rat. The ligation of the left anterior descending branch of the left coronary artery was covered with the gauze after the wetting of the saline. After half an hour, the ligature was cut through with surgical scissors to restore the blood flow to the reperfusion of the coronary artery. SD rats were sacrificed after $3 \mathrm{~h}$ making the model, and the damaged parts of the heart were selected. Formalin solution was used to fixing and paraffin-embedded section was carried out. Then the immunochemistry and TUNEL were performed. Only those with thread without ligation were the sham operation group, and no animals died during the ligation and reperfusion.

Detection of arrhythmia. Referring to the previous scoring methods for ventricular arrhythmias (VA) (6), the score of VE with no VA or $<5$ times is 0 points, and the score of VE with $\geq 5$ times is 1 point. $\mathrm{T}$ at a time $<60 \mathrm{sec}$ is 2 points, $\mathrm{VT}$ at a time $\geq 60 \mathrm{sec}$ or multiple accumulation $<60 \mathrm{sec}$ is 3 points, and multiple accumulation $>60 \mathrm{sec}$ is 4 points. VT occurrence is 5 points, VF occurrence continuously over $5 \mathrm{~min}$ or death during the observation period is 6 points.

Detection of myocardial infarction area. The left ventricle was sliced to a thickness of $\sim 2 \mathrm{~mm}, 5$ pieces in total, and put in a $0.25 \% \mathrm{NBT}$ dyeing solution to stain at $37^{\circ} \mathrm{C}$ for $15 \mathrm{~min}$. At the time of necrosis of the myocardium, gray white could be seen, while the non-necrotic myocardium was blue. The water was absorbed with clean absorbent paper, the necrotic area of the myocardium was slowly cut down and weighed. The weight of the necrotic region of the myocardium was recorded, and the proportion of the myocardial weight in the myocardial infarction area accounted for the weight of the left ventricle was calculated.
Immunohistochemical staining. Immunohistochemical examination was performed according to the manufacturer's instructions. Paraffin-embedded treatment was carried out on myocardial tissue block (fixed with $4 \%$ polyformaldehyde at $4^{\circ} \mathrm{C}$ overnight), paraffin section dewaxing, hydration, PBS washing. After blocking for $15 \mathrm{~min}, 10 \%$ serum at room temperature was blocked in non-specific background for 15 min, anti-caspase-3 monoclonal antibody was added (dilution, 1:200; cat. no. 9662; Cell Signaling Technology, Inc.), and stored in a refrigerator at $4^{\circ} \mathrm{C}$ overnight. Rinsing by PBS after taking out the horseradish peroxidase (HRP) labeled secondary antibody (dilution, 1:5,000; cat. no. 3875; Cell Signaling Technology, Inc.) the biotin labeled secondary antibody was added, then incubated at room temperature for $30 \mathrm{~min}$ before rinsing. Streptomyces antibiotic protein peroxidase solution was incubated at room temperature for $30 \mathrm{~min}$ and then washed again. After DAB staining, rinsing, redying, sealing, and observing under an optical microscope (LSM880; Carl Zeiss AG with Airyscan, Oberkochen, Germany), the cytoplasm of caspase-3 positive cells were brownish or brown color.

Positive cell rate $\%=$ positive cells count/(positive cells count + negative cells count) $\times 100 \%$.

Detection of apoptosis by TUNEL. Strictly referring to the operation procedure of one step TUNEL apoptosis in situ assay kits, the tissue block was rinsed with dimethylbenzene and gradient alcohol, and then rinsed and dried after incubating proteinase $\mathrm{K}$ at room temperature, and added $50 \mu \mathrm{l}$ TUNEL liquid, rinsed after incubating in a dark wet box at room temperature for 1 hour, and the $50 \mu \mathrm{l}$ converted-POD was added, and incubated for another $30 \mathrm{~min}$. After washing, $100 \mu \mathrm{l}$ DAB substrate was added and incubated for $10 \mathrm{~min}$ at room temperature and then rinsed, redyed and dehydrated. After rinsing with xylene, it was sealed and observed under the microscope. Under the optical microscope, it was found that there were brown granules in cytoplasm and nucleus, which was a positive expression. In the positive cell distribution area, 5 different visual fields were randomly selected. The average number was used as the quantitative standard of apoptosis, and the percentage of positive cells was calculated.

Detection of expression of caspase-3 by western blotting. The protein was extracted according to the steps in the tissue protein extraction instructions. The supernatant was taken as total protein after centrifugation at $12,000 \mathrm{x} \mathrm{g}$ for $10 \mathrm{~min}$ at $4^{\circ} \mathrm{C}$. The protein was quantified by BCA protein quantitative kit (Pierce; Thermo Fisher Scientific, Inc., Waltham, MA, USA). Sampling buffer system with equal concentration was prepared and boiled at $95^{\circ} \mathrm{C}$ for $15 \mathrm{~min}$. A total of $10 \%$ SDS-PAGE gel was placed and each pore was $15 \mu \mathrm{l}$. Then, $100 \mathrm{~V}$ was used for electrophoresis. After the end of electrophoresis, the protein was transferred to a PVDF membrane by wet method. The membrane was transferred for $2 \mathrm{~h}(90 \mathrm{~V})$, and then it was immersed in the closed liquid, and placed on the rocking bed for $2 \mathrm{~h}$. The caspase-3 (dilution, 1:1,000; cat. no. 9662; Cell Signaling Technology, Inc.) and $\beta$-actin primary antibody (dilution, 1:1,000; cat. no. 3700; Cell Signaling Technology, Inc.) was added, then stored in a refrigerator at $4^{\circ} \mathrm{C}$ overnight, and $\beta$-actin was used as the internal reference. TBST was 


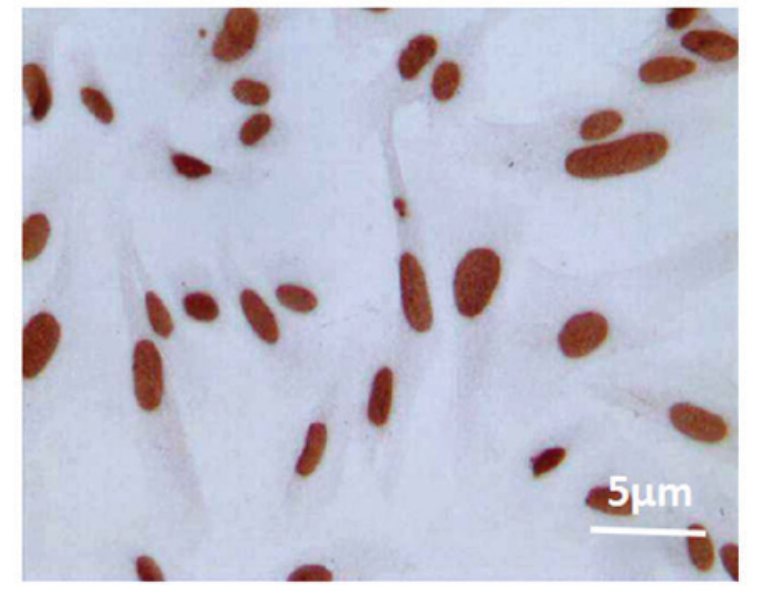

Figure 1. The positive staining diagram of apoptosis (magnification, $\mathrm{x} 100$ ).

Table I. The myocardial infarction area of SD rats after ischemia reperfusion (mean \pm standard deviation).

\begin{tabular}{lcc}
\hline Groups & No. of cases & $\begin{array}{c}\text { Myocardial infarction } \\
\text { range (\%) }\end{array}$ \\
\hline Blank & 12 & 0 \\
Sham operation & 12 & 0 \\
Ischemia reperfusion & 12 & $30.16 \pm 2.53^{\mathrm{a}}$ \\
Simvastatin & 12 & $23.95 \pm 1.89^{\mathrm{a}, \mathrm{b}}$ \\
\hline
\end{tabular}

${ }^{\mathrm{a}} \mathrm{P}<0.001$, compared with the blank and sham operation groups. ${ }^{\mathrm{b}} \mathrm{P}=0.007$, compared with the ischemia-reperfusion group.

washed for $10 \mathrm{~min}$ at room temperature 3 times. Horseradish enzyme labeled sheep against rabbit IgG antibody (dilution, 1:5,000; cat. no. 3875; Cell Signaling Technology, Inc.) was added and incubated for $1 \mathrm{~h}$ at room temperature and then, TBST was washed for $10 \mathrm{~min}$ at room temperature 5 times. Fresh ECL luminescent liquid (Cell Signaling Technology Inc.; mixed with liquids A and B) was added to a PVDF film to develop in the dark. After chromogenic photography, the strips obtained were processed and analyzed by ImageJ software (ImageJ2x; Rawak Software, Inc., Dresden, Germany).

Statistical analysis. The SPSS 19.0 software (SPSS Inc., Chicago, IL, USA) was used to analyze the data, and the measurement data were expressed as mean \pm standard deviation (mean $\pm \mathrm{SD}$ ). Analysis of variance (ANOVA) was used for comparison among groups, and the Bonferroni method was used for comparison between two groups if the variance was homogeneous, while the Welch's method was used for analysis if the variance was not homogeneous. $\mathrm{P}<0.05$ was considered to be statistically different.

\section{Results}

Staining diagram of positive apoptosis. Under the optical microscope, it was found that there were brown granules in cytoplasm and nucleus, indicating that the cells in the myocardium were apoptotic (Fig. 1).

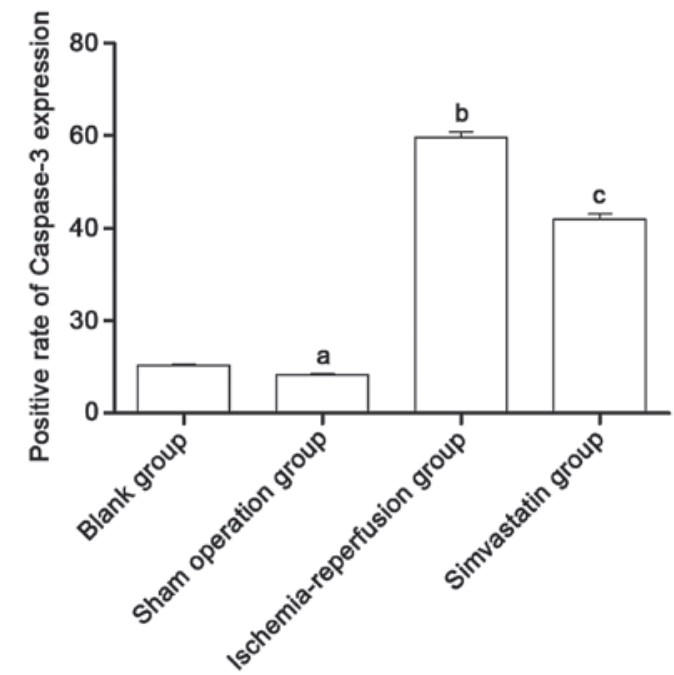

Figure 2. The positive rate of expression of caspase-3. ${ }^{\mathrm{a}} \mathrm{P}=0.041$, compared with the blank group. ${ }^{b} \mathrm{P}<0.001$, compared with the sham operation group. ${ }^{\mathrm{c}} \mathrm{P}=0.027$, compared with the ischemia-reperfusion group.

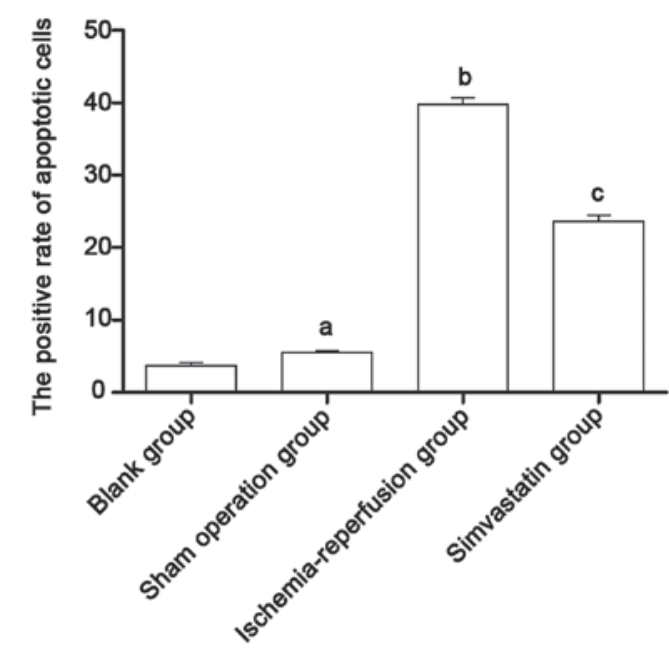

Figure 3 . The positive rate of apoptotic cells. ${ }^{\text {a }} \mathrm{P}=0.044$, compared with the blank group. ${ }^{b} \mathrm{P}<0.001$, compared with the sham operation group. ${ }^{c} \mathrm{P}=0.018$, compared with the ischemia-reperfusion group.

Effect of ischemia-reperfusion on infarct area in SD rats. NBT staining showed that the myocardium of SD rats in the blank and sham operation groups were all stained blue, and no gray white was seen. The myocardium of SD rats in the ischemia reperfusion and simvastatin groups showed a more obvious gray white infarct area which in the simvastatin group was significantly smaller than the ischemia reperfusion group. The difference between the two groups was statistically significant $(\mathrm{P}=0.007)$ (Table I).

Effect of simvastatin on expression of caspase-3 in SD rats. The expression of caspase- 3 in the ischemia-reperfusion group was significantly higher than that in the sham operation group $(\mathrm{P}<0.001)$. The expression of caspase- 3 in the simvastatin group SD rats was significantly lower than the expression of caspase-3 in the SD rats of the ischemia reperfusion group, and the difference between the two groups was significant $(\mathrm{P}=0.027)$ (Fig. 2). 
Table II. The score of arrhythmia (mean \pm standard deviation).

\begin{tabular}{lcc}
\hline Groups & No. of cases & Arrhythmia score \\
\hline Blank & 12 & 0 \\
Sham operation & 12 & 0 \\
Ischemia reperfusion & 12 & $4.2 \pm 1.5^{\mathrm{a}}$ \\
Simvastatin & 12 & $3.1 \pm 2.3^{\mathrm{b}}$ \\
\hline
\end{tabular}

${ }^{\mathrm{a}} \mathrm{P}<0.001$, compared with the blank and sham operation groups. ${ }^{\mathrm{b}} \mathrm{P}=0.033$, compared with the ischemia-reperfusion group.
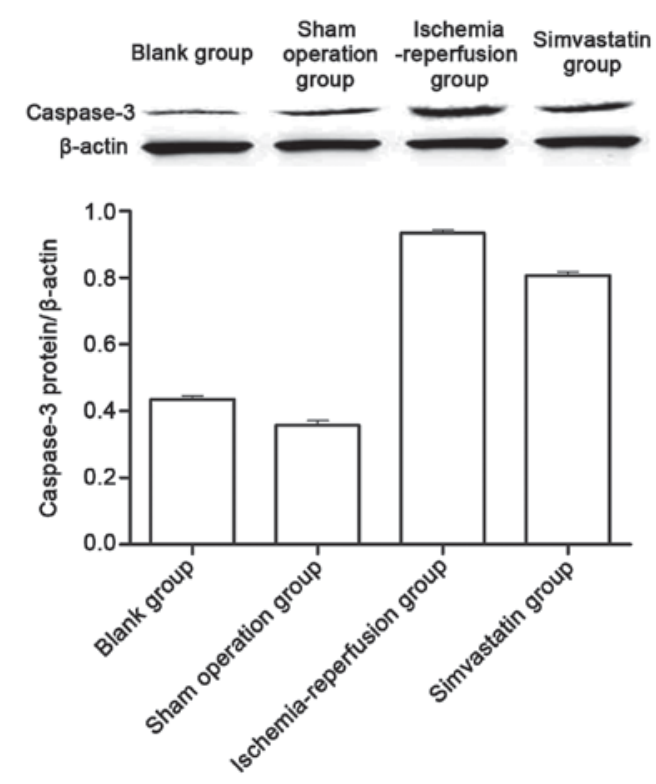

Figure 4 . The detection of the expression of caspase- 3 protein by western blotting.

Effect of simvastatin on apoptotic cells in SD rats. Apoptosis was detected in a very small number of cells both in the blank group and the sham operation group. There was a large number of apoptotic cells in the ischemia reperfusion and simvastatin groups, and the number of apoptotic cells in the simvastatin group SD rats was significantly lower than the number of apoptotic cells in the SD rats of the ischemia reperfusion group. The difference between the two groups was statistically significant ( $\mathrm{P}=0.018)$ (Fig. 3).

Reperfusion arrhythmia monitoring. Arrhythmia in SD rats was not found in the blank and sham operation groups, and the arrhythmia score was 0 , while arrhythmia in SD rats was observed in the ischemia reperfusion and simvastatin groups, and the arrhythmia score of the simvastatin group was significantly lower than the arrhythmia score of the ischemia reperfusion group, and the two groups were evaluated. The difference was statistically significant $(\mathrm{P}=0.033)$ (Table II).

Detection of expression of caspase-3 protein by western blotting. Compared with the blank and sham operation groups, the expression of caspase-3 protein in the ischemia-reperfusion and simvastatin groups was significantly higher than the simvastatin group, and the expression of caspase-3 protein in the simvastatin group was significantly lower than the expression of caspase- 3 protein in the ischemia-reperfusion group. The difference was statistically significant $(\mathrm{P}=0.038)$ (Fig. 4).

\section{Discussion}

Four types of cardiac dysfunction occurred after myocardial ischemia reperfusion injury, including reflux, myocardial stunning, fatal reperfusion injury and reperfusion arrhythmia $(7,8)$. At present, there are different explanations for the mechanism of myocardial ischemia-reperfusion injury, such as oxygen free radical theory (9), calcium overload theory (10) and inflammatory response theory (11). Kocak et al (12) suggested that apoptosis would occur during myocardial ischemia-reperfusion injury, and apoptosis plays a decisive role in the final infarct area of myocardium. When a specific drug is used to intervene in the process of apoptosis, the effective inhibition of apoptosis can make the area of the myocardial infarction area up to $70 \%$, significantly improving the state of heart function (13). Therefore, the use of anti-apoptotic drugs in clinical treatment of myocardial ischemia reperfusion injury have a great significance.

Apoptosis is also known as programmed cell death (PCD), and caspase-3 is a very important starting factor (14), which mediates PCD process. It has been shown that caspase- 3 can be activated by its own cleavage by oligomerization, and can activate a variety of downstream proteases, such as cysteine protease, and participate in the process of inhibiting cell proliferation and inducing cell apoptosis (15). Zheng et al (16) found that caspase-3 inhibitors can effectively inhibit the apoptosis of rabbit cardiopulmonary bypass cardiomyocytes. It is presumed that it may be associated with the inhibition of the activity of caspase-3. Wang et al (17) found that the use of different doses of atorvastatin preconditioning acute myocardial ischemia animal model, can significantly reduce apoptosis of myocardial cells and inhibit the expression of caspase-3 gene. Huang et al (18) found that atorvastatin can inhibit apoptosis of myocardial cells in rats with heart failure after myocardial infarction, and then reduce the structural reconstruction of the heart and improve the cardiac function.

In this study, the expression of caspase-3 was significantly increased in the ischemia-reperfusion and simvastatin groups, which was mainly distributed in the gray white region, indicating that the cardiomyocytes of the SD rats were obviously apoptotic after ischemia-reperfusion, and apoptosis of the simvastatin group was significantly lower than that of the ischemia reperfusion group $(\mathrm{P}<0.05)$; the positive rate of caspase-3 was decreased $(\mathrm{P}<0.05)$, the area of myocardial infarction area was decreased $(\mathrm{P}<0.05)$ and the rate of arrhythmia was significantly decreased $(\mathrm{P}<0.05)$. The effect of myocardial apoptosis in the simvastatin group was significantly inhibited, and the incidence of cardiac infarction and arrhythmia was reduced, so as to exert a better protective effect on myocardium, which is in accordance with the results of Ma et al (19). At present, it is not known how simvastatin can protect the myocardium by reducing the expression of caspase- 3 through anti-apoptosis. However, Liu et al (20) indicated that the expression of p53 and caspase- 3 in simvastatin decreased significantly in the the simvastatin group with simvastatin preconditioning renal ischemia reperfusion, and the level of oxygen free radicals 
were also decreased $(\mathrm{P}<0.05)$. It is presumed that simvastatin may scavenge the excess oxygen free radicals induced by renal ischemia-reperfusion injury, and then decrease the oxidative damage effect of myocardial cells, reduce the expression of p53 and caspase-3, and ultimately inhibit cell apoptosis. However, for the role of myocardial ischemia reperfusion injury, we need to further design experiments for verification.

In conclusion, simvastatin has a protective effect on myocardial ischemia reperfusion injury, and its mechanism may be associated with reducing the expression of caspase-3 and inhibiting apoptosis.

\section{Acknowledgements}

Not applicable.

\section{Funding}

No funding was received.

\section{Availability of data and materials}

The datasets used and/or analyzed during the present study are available from the corresponding author on reasonable request.

\section{Authors' contributions}

WS conceived and designed the study. RP and JS were responsible for the construction of the ischemia-reperfusion injury model. WS and HS detected arrhythmia and myocardial infarction area. All authors read and approved the final manuscript.

\section{Ethics approval and consent to participate}

The study was approved by the Ethics Committee of Yancheng TCM Hospital Affiliated to Nanjing University of Chinese Medicine (Yancheng, China).

\section{Patient consent for publication}

Not applicable.

\section{Competing interests}

The authors declare that they have no competing interests.

\section{References}

1. Sopková J, Vidomanová E, Strnádel J, Škovierová H and Halašová E: The role of statins as therapeutic agents in cancer. Gen Physiol Biophys 36: 501-511, 2017.

2. Nykänen AI, Tuuminen R and Lemström KB: Donor simvastatin treatment and cardiac allograft ischemia/reperfusion injury. Trends Cardiovasc Med 23: 85-90, 2013.

3. Akarsu M, Saygun O, Aydinuraz K, Aydin O, Daphan CE, Tanrikulu FB, Kisa U and Comu FM: The effects of simvastatin on ischemia reperfusion injury in an experimental colon anastomosis model. Indian J Surg 79: 390-395, 2017.
4. Zhang Y, Zhang Z and Yan H: Simvastatin inhibits ischemia/reperfusion injury-induced apoptosis of retinal cells via downregulation of the tumor necrosis factor- $\alpha /$ nuclear factor- $\kappa \mathrm{B}$ pathway. Int J Mol Med 36: 399-405, 2015.

5. Hadi NR, Al-Amran F, Yousif M and Zamil ST: Antiapoptotic effect of simvastatin ameliorates myocardial ischemia/reperfusion injury. ISRN Pharmacol 2013: 815094, 2013.

6. Huang LH, Li J, Gu JP, Qu MX, Yu J and Wang ZY: Butorphanol attenuates myocardial ischemia reperfusion injury through inhibiting mitochondria-mediated apoptosis in mice. Eur Rev Med Pharmacol Sci 22: 1819-1824, 2018.

7. Zhou T, Guo S, Wang S, Li Q and Zhang M: Protective effect of sevoflurane on myocardial ischemia-reperfusion injury in rat hearts and its impact on HIF-1 $\alpha$ and caspase-3 expression. Exp Ther Med 14: 4307-4311, 2017.

8. Zhao Y, Feng Q, Huang Z, Li W, Chen B, Jiang L, Wu B, Ding W, Xu G, Pan H, et al: Simvastatin inhibits inflammation in ischemia-reperfusion injury. Inflammation 37: 1865-1875, 2014.

9. Al-Herz W and Babiker F: Acute intravenous infusion of immunoglobulins protects against myocardial ischemia-reperfusion injury through inhibition of caspase-3. Cell Physiol Biochem 42: 2295-2306, 2017.

10. Hwang J, Han JI and Han S: Effect of pretreatment with simvastatin on spinal cord ischemia-reperfusion injury in rats. J Cardiothorac Vasc Anesth 27: 79-85, 2013.

11. Tuuminen R, Holmström E, Raissadati A, Saharinen P, Rouvinen E, Krebs R and Lemström KB: Simvastatin pretreatment reduces caspase-9 and RIPK1 protein activity in rat cardiac allograft ischemia-reperfusion. Transpl Immunol 37: 40-45, 2016.

12. Kocak FE, Kucuk A, Ozyigit F, Tosun M, Kocak C, Kocak A, Ekici MF, Yaylak F and Genc O: Protective effects of simvastatin administered in the experimental hepatic ischemia-reperfusion injury rat model. J Surg Res 199: 393-401, 2015.

13. Song L, Gao LN, Wang J, Thapa S, Li Y, Zhong XB, Zhao HW, Xiang XR, Zhang FG and Ji P: Stromal cell-derived factor- $1 \alpha$ alleviates calcium-sensing receptor activation-mediated ischemia/reperfusion injury by inhibiting caspase-3/caspase-9-induced cell apoptosis in rat free flaps. BioMed Res Int 8945850: 2018, 2018.

14. Han QF, Wu L, Zhou YH, Wang LH, Zhang DY, Liu T and Yao HC: Simvastatin protects the heart against ischemia reperfusion injury via inhibiting HMGB1 expression through PI3K/Akt signal pathways. Int J Cardiol 201: 568-569, 2015.

15. Yi X, Cui X, Wu P, Wang S, Wang G, Yang X, Yang F, Zheng S and Li Z: Effects of $\mathrm{N}$-acetylcysteine on apoptosis induced by myocardial ischemia reperfusion injury in rats' heart transplantation. Zhongguo Xiu Fu Chong Jian Wai Ke Za Zhi 27: 1234-1239, 2013 (In Chinese).

16. Zheng C, Wu Z, Tian L, Li D, Wang X, He Y, He Y, Jin W, Li M, Zhu Q, et al: Long noncoding RNA AK12348 is involved in the regulation of myocardial ischaemia-reperfusion injury by targeting PARP and caspase-3. Heart Lung Circ 27: e51-e58, 2018.

17. Wang SY, Cui XL, Xue FS, Duan R, Li RP, Liu GP, Yang GZ and Sun C: Combined morphine and limb remote ischemic perconditioning provides an enhanced protection against myocardial ischemia/reperfusion injury by antiapoptosis. J Surg Res 202: 13-25, 2016.

18. Huang $\mathrm{CH}$, Lai CC, Yang $\mathrm{AH}$ and Chiang SC: Myocardial preconditioning reduces kidney injury and apoptosis induced by myocardial ischaemia and reperfusion. Eur J Cardiothorac Surg 48: 382-391, 2015.

19. Ma J, Qiao Z and Xu B: Effects of ischemic preconditioning on myocardium Caspase-3, SOCS-1, SOCS-3, TNF- $\alpha$ and IL-6 mRNA expression levels in myocardium IR rats. Mol Biol Rep 40: 5741-5748, 2013.

20. Liu K, Chen H, You QS, Ye Q, Wang F, Wang S, Zhang SL, Yu KJ and $\mathrm{Lu} \mathrm{Q}$ : Curcumin attenuates myocardial ischemia-reperfusion injury. Oncotarget 8: 112051-112059, 2017.

This work is licensed under a Creative Commons Attribution-NonCommercial-NoDerivatives 4.0 International (CC BY-NC-ND 4.0) License. 